\title{
An Example of PCP-DDS Collaboration in DSM
}

\author{
J. Michael Adame, DDS, D,ABDSM¹ , Joel Solis, MD ${ }^{2,3}$
}

\begin{abstract}
${ }^{1}$ Adame Dental Sleep Medicine, ${ }^{2}$ University of Texas Medical Branch, Galveston, TX, ${ }^{3}$ University of Texas Rio Grande Valley Family Medicine Residency, McAllen, TX
\end{abstract}

The medical and dental patients in our region of south Texas are afflicted by multiple, chronic diseases including hypertension, congestive heart failure, and diabetes. Many of these patients also suffer from obstructive sleep apnea (OSA). Their health challenges are compounded by the fact that our community is medically underserved. Because both of us are eager to improve outcomes for these patients, it was natural for us to begin collaborating on using oral appliance therapy to treat OSA.

Our collaboration began a few years ago. In that time, we've worked together to treat between 40 and 50 patients. This is the story of how that collaboration came to be, the effect it has had on our patients, and how it can evolve to be a model for treatment of OSA and other chronic diseases.

Joel Solis, MD (JS): As a physician, I'm always looking for new treatments to better help my patients, especially with complex conditions such as OSA. I was aware of positive airway pressure (PAP) therapy as a treatment option, but some of my patients did not like it or were unwilling to try it. One day, I got a call from Dr. Michael Adame, a local dentist who is a Diplomate of the American Board of Dental Sleep Medicine and who treats OSA with oral appliance therapy. He told me that one of our mutual patients wanted to try an oral appliance for her OSA, so I jumped!

Michael Adame, DDS (MA): The patient had recently asked me about oral appliances for OSA during a routine visit because she was looking for a treatment other than PAP. I told her that a physician must prescribe an oral appliance. Dr. Solis was her primary care physician. From talking to her, I could tell that she trusted and respected Dr. Solis, so I called him.

JS: I wanted my staff and I to learn more about oral appliance therapy, so I invited Dr. Adame to teach us more about it. From there we began to develop methods of collaboration with the goal of bringing about the best outcomes for our mutual patients. Now, we regularly touch base about each patient, discussing the status of their treatment and the results the patient is experiencing. From there, we decide what next steps will be appropriate. Although we do use email, two crucial parts of the process are our regularly scheduled phone calls and face-to-face meetings, which allow us to cover the details and nuances necessary to ensure that each patient is getting the maximum benefit from OAT. In five or six cases, the two of us have even met with a patient at the same time.

MA: The American Academy of Dental Sleep Medicine (AADSM) has developed a model for collaboration between physicians and dentists, and this model guides our work (Figure 1). According to the model, the physician is solely responsible for diagnosis and evaluation of the patient's overall health. The dentist ensures that the oral appliance fits correctly and is treating the symptoms of OSA while watching for any dental side effects. The dentist can adjust the device to optimize the outcomes and will refer the patient back to the primary care physician whenever it is warranted to do so. In the model we have established, we also work with board-certified sleep medicine physicians who interpret the results of home sleep apnea tests during the diagnosis process and to verify treatment efficacy.

JS: Although it might seem that so many regular conversations about our patients would take too much time away from our daily routines, they actually save time in the long run. Collaboration enhances continuity of care, which is pivotal to treatment effectiveness. Not surprisingly, my outcomes are extremely good when I work with another clinician.

MA: Our collaboration is iterative, and we are always finding new and better ways to work together. OSA is a chronic condition, so we are always looking years down the road. We set up the path for patients to follow, and our back-and-forth conversations allow us to make better adaptions in the future.

JS: Patients don't know why we wouldn't talk to each other. The earlier we make connections to other clinicians, the more treatment improves. If I can have even just one face-to-face encounter with another clinician, it hugely benefits the outcomes.

MA: When patients know that we are talking to each other about their treatment, they own their care to a much greater extent. They keep their appointments, and they adhere to the treatment protocols. It's like when two rivers come together, and the confluence makes the water move faster. Our collaboration moves the patient toward health more quickly.

JS: One patient who illustrates our collaboration is $\mathrm{Mr}$. K. He is one of my patients who suffers from diabetes. During one of his regular checkups, he asked, "Can you 
Figure 1. Clinical Pathway for the Management and Treatment of SRBD

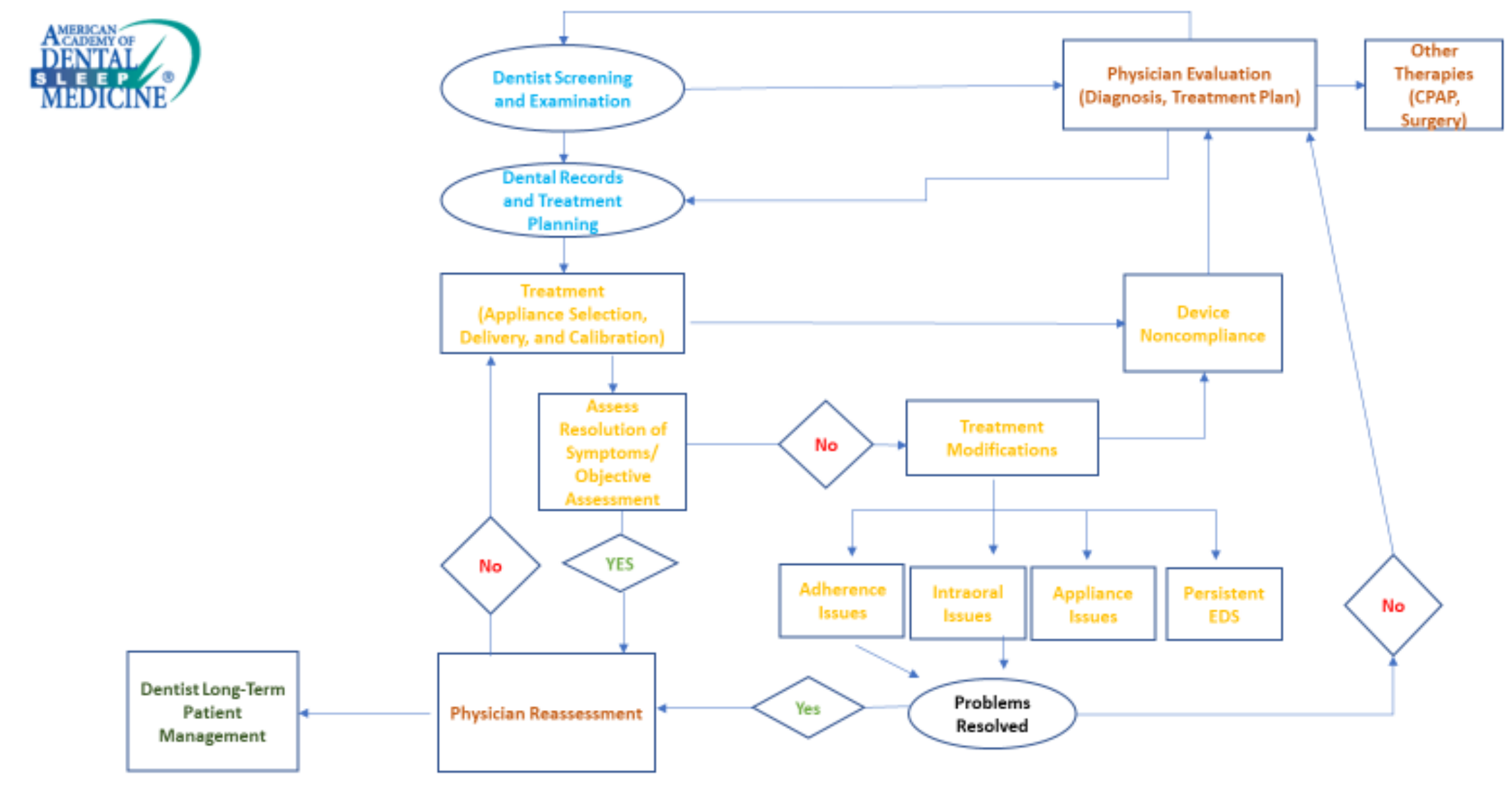

CPAP = Continuous Positive Airway Pressure; EDS = Excessive Daytime Sleepiness

Levine M, Bennett K, Cantwell M, Postol K, Schwartz, D. Dental sleep medicine standards for screening, treating and managing adult patients with sleep-related breathing disorders. J Dent Sleep Med. 2018;5(3):61-68 
help me get off the couch? I've been sleeping on it for 6 months!" We discussed his symptoms, which were the classic symptoms of OSA. I ordered a home sleep apnea test and when I got the interpreted results back from the board-certified sleep medicine physician, Mr. K was diagnosed with OSA, prescribed oral appliance therapy, and referred to Dr. Adame.

MA: I evaluated his teeth and his airway to make sure that he would be a good candidate for an oral appliance. Next, I took impressions and sent them to a laboratory where the oral appliance was fabricated. He returned to my office and I fitted him with the appliance. I then sent a letter to Dr. Solis thanking him for the referral. It's my standard protocol to include several key facts in my follow-up letters to primary care physicians: that the patient was a good candidate for oral appliance therapy, the date on which the oral appliance was delivered, that I will be following up with the patient over the course of up to 12 weeks, and that I will continue to keep the physician updated.

During my follow-ups, I collect subjective data and compare these data to the preoperative baselines that I had obtained. After 6weeks, Mr. K reported that he was no longer sleeping on the couch, that his energy level was much higher, and he had moved from a score of 9 on the Epworth Sleepiness Scale down to a score of 3, indicating a positive result. At this point, I usually send a verification letter to the physician, reporting the results that I have seen and indicating that the patient is ready for the physician to verify that the oral appliance is managing the OSA.

For the patients whom Dr. Solis and I share, he orders the home sleep apnea test that I then administer to the patient. The results of the home sleep apnea tests are interpreted by a board-certified sleep medicine physician. As we get the results of the home sleep apnea tests, I may adjust the oral appliance to ensure that it is in its optimal position. Dr. Solis then uses the interpretation of the home sleep apnea test and subjective data to verify that his patients are being effectively treated. In this case, Mr. K. went from an apnea-hypopnea index of 30 events/h (severe sleep apnea) down to 2 events/h (no sleep apnea) and was feeling much better.

JS: This patient also felt good about the overall course of treatment because we did our best to make sure that he did not need to have multiple visits. It's hard to ask that of a patient, and I need Dr. Adame's help to keep the number of appointments to a minimum. We maintain a line of communication by phone if we have time, but if not, we use email. My electronic medical record will put his messages into a bin that I am responsible for reviewing. My authorization is then required to proceed with treatment, much like a physical therapy referral. For follow-up, I try to see all OSA patients every 3 months.

We've also extended the collaboration model to our respective staff. They meet with each other to discuss the treatment status and next steps for our mutual patients.

JS: Although our time with each patient is limited, I strongly believe that all primary care physicians should start screening their patients for OSA. When Mr. K. asked me to help him get off the couch, he wasn't there to be screened for sleep apnea. Now, he's back to sleeping in his bed and getting the rest he needs to prevent more serious comorbidities down the road.

MA: Dentists can be screening our patients too. We can find at least one patient every day who needs additional care and refer them to their primary care physician for further evaluation.

JS: As a physician, I want to be great at diagnosis, and I want to know about all possible treatment options. Unfortunately, many primary care physicians don't understand OSA, and it is critical that we learn more. OSA is a monster disease - it has a severe effect on multiple biological systems, resulting in multiple, chronic comorbidities. We are at risk of misdiagnosis if we don't understand it, and we might end up prescribing a therapy that won't work.

We also need to understand the full range of treatment options for OSA and how effective they are. As doctors, we always think of PAP first. However, a lot of patients don't want to try PAP. Fortunately, there are other options. One option is oral appliance therapy - that's where dentists come in, because they know more about it than we do.

MA: However, not all dentists have been trained in dental sleep medicine, and primary care physicians should ensure that they establish relationships with qualified dentists. This includes dentists who have earned the AADSM Qualified Dentist designation and dentists who are Diplomates of the American Board of Dental Sleep Medicine. The Find-an-AADSM-Dentist locator at aadsm.org is the best way to find one near you. These trained dentists are eager to work with primary care physicians in a model of care that works for the physician and provides optimal patient care.

JS: A team approach is necessary to improve patient outcomes for chronic diseases such as OSA and diabetes. The severity and prevalence of these diseases are straining our ability to provide care. In addition, business pressures are forcing us to find more efficient and effective treatment methods. Collaboration allows us to provide better solutions faster. It is what our patients need, and it is what we need to take the strain off our days.

\section{ABOUT THE AUTHORS}

J. Michael Adame, DDS, D.ABDSM is a Diplomate of the American Board of Dental Sleep Medicine with offices in McAllen, Edinburg and Donna, Texas. Dr. Adame graduated from the University of Texas Health Science Center of San Antonio in 1987 and has been involved in dental sleep medicine for over 10 years.

Joel Solis, MD is a board-certified family medicine physician in McAllen, Texas. Dr. Solis graduated from the University of Medicine \& Dentistry of New Jersey Robert 
Wood Johnson Medical School in 1999 and has been in practice for 17 years. He is adjunct faculty at University of Texas Medical Branch in Galveston and at University of Texas Rio Grande Valley Family Medicine Residency in McAllen.

\section{CITATION}

Adame M, Solis, J. How dentists can help you manage your OSA caseload. J Dent Sleep Med. 2020;7(1)

\section{SUBMISSION AND CORRESPONDENCE INFORMATION}

Submitted in final revised form November 12, 2019 Accepted for publication November 12, 2019

Address correspondence to: J. Michael Adame, DDS, D.ABDSM, 206 W. Mahl, Edinburg, Texas 78539; Email: jma@jmichaeladamedds.com

\section{DISCLOSURE STATEMENT}

The authors report no conflicts of interest. 